\title{
EVALUACIÓN DEL COMPLEJO VACUNAL ANTÍGENO-ANTICUERPO DE LA ENFERMEDAD DE GUMBORO ADMINISTRADO in ovo EN POLLOS DE CARNE FRENTE A UN DESAFÍO EXPERIMENTAL CON LA CEPA F52/70
}

\author{
Evaluation of the Antigen-Antibody Complex Vaccine of Gumboro Disease \\ Administered in ovo in Broiler Chickens Challenged with F52/70 Strain
}
John Guzmán G. ${ }^{1}$, Mónica Alba Ch. ${ }^{1}$, Eliana Icochea D. ${ }^{1,2}$, Alberto Manchego S. ${ }^{3}$, Rosa Perales C. ${ }^{4}$, Susana Fribourg C. ${ }^{5}$

\section{Resumen}

\begin{abstract}
El objetivo del presente estudio fue evaluar la protección y seguridad del complejo vacunal antígeno-anticuerpo administrado in ovo frente a un desafío experimental con la cepa F52/70 de la Enfermedad de Gumboro en pollos de carne. Se utilizaron 450 pollos de la línea Cobb Vantress, de un día de edad, distribuidos en tres grupos: Grupo A, vacunado, vía agua de bebida, a los 9 días con la cepa intermedia suave tipo Lukert y revacunado a los 19 días con la cepa intermedia intermedia 2512; grupo B, vacunado vía in ovo a los 18 días de incubación con el complejo vacunal antígeno-anticuerpo; y grupo C, control no vacunado. A los 35 días de edad, 20 aves de cada grupo fueron desafiadas, vía ocular, con la cepa F52/70 de la enfermedad de Gumboro. La seguridad del complejo vacunal se evaluó semanalmente hasta los 47 días de edad mediante la determinación del índice bursal, lesiones macroscópicas, lesiones histopatológicas de bursa y respuesta serológica. Las lesiones post desafío se caracterizaron por edema y hemorragias petequiales en la bursa. El grupo B presentó la mejor protección con $75 \%$ comparado con el grupo A (68\%) y el grupo control (30\%). Las lesiones post desafío se caracterizaron por edema y hemorragias petequiales en la bursa. En las aves no desafiadas no se observó diferencias significativas entre los grupos A y B para el índice bursal y lesiones histopatológicas hasta los 28 días de edad; sin embargo, a partir de los 35 días el grupo A fue significativamente diferente de los grupos B y C ( $\mathrm{p}<0.05)$. Al final del estudio el grupo A obtuvo títulos de 1948 y 2047 más de anticuerpos que el grupo B y el control, respectivamente.
\end{abstract}

Palabras clave: complejo vacunal antígeno-anticuerpo, enfermedad de Gumboro, cepa 2512, índice bursal, atrofia bursal, edema, depleción linfoide, respuesta humoral, respuesta celular

\footnotetext{
${ }^{1}$ Laboratorio de Patología Aviar, ${ }^{3}$ Laboratorio de Microbiología y Parasitología Veterinaria, ${ }^{4}$ Laboratorio de Embriología, Histología, y Patología Veterinaria, Facultad de Medicina Veterinaria, Universidad Nacional Mayor de San Marcos, Lima

${ }^{2}$ E.mail: eliana.icochea@gmail.com

${ }^{5}$ AVEAGRO S.A., Lima
} 
The objective of the study was to evaluate the protection and safety for in ovo vaccination against Infection Bursal Disease. Cobb Vantress broilers of one day of age $(n=450)$ were distributed in three groups: Group A, vaccinated at 9 and 19 days with two commercial live vaccines containing Lukert and 2512 strains, respectively; group B, vaccinated in ovo with the antigen-antibody complex at 18 days of incubation; and group $\mathrm{C}$, unvaccinated. At 35 days of age, 20 birds from each group were challenged, ocular via, with the F52/70 strain of Gumboro disease. The security of the complex vaccine was evaluated weekly until 47 days of age by determining the bursal index, gross lesions, histopathological lesions of the bursa, and serological response. Group B showed the best protection with $75 \%$ as compared with groups A (68\%) and C (30\%). Injuries after challenge were characterized by swelling, bleeding, and petechiae in the bursa. In non-challenged birds were none significant differences between groups A and B for the index and bursal histopathology lesions until 28 days of age; however, group A differed from groups B and C at 35 days of age $(\mathrm{p}<0.05)$. At the end of the study, group A had 1948 and 2047 antibodies titers higher than groups B and control respectively.

Key words: antigen-antibody complex vaccine, Gumboro diseases, 2512 strain, bursal index, bursal atrophy, edema, lymphoid depletion, humoral response, cellular response

\section{INTRODUCCIÓN}

La Enfermedad Infecciosa de la Bursa (EIB) es una amenaza permanente para la industria avícola, debido a las repercusiones en el rendimiento económico de los planteles de reproductoras y pollos de carne. La EIB es considerada como la principal causa de inmunosupresión, ocasionando un incremento en la susceptibilidad de las aves domésticas a otros agentes infecciosos como la enfermedad de Newcastle, Bronquitis Infecciosa, Marek, e infecciones bacterianas (Rosales, 2000; Montiel, 2004).

La enfermedad es de distribución mundial, con mayor incidencia en las grandes áreas de producción avícola; es altamente contagiosa y afecta a los pollos hasta las 6 semanas de edad causando una morbilidad de hasta $100 \%$ y una mortalidad que varía de 20 a 30\%. La forma subclínica es la más importante, se presenta en aves hasta las tres semanas de edad, y aunque no presenta signos clínicos evidentes, se caracteriza por una severa inmunodepresión que se manifiesta en la incidencia de otras enfermedades. La forma clínica se observa en aves mayores de 3 semanas, de aparición súbita con mortalidad elevada a los 5 a 7 días post infección, y se caracteriza por diarrea, decaimiento y deshidratación, seguida de una rápida recuperación de los sobrevivientes (Lukert y Saif, 2003).

El control y prevención de la enfermedad de Gumboro continúa siendo un desafío en la producción avícola y depende de la implementación de un programa integral de bioseguridad, vacunación y diagnóstico serológico (Montiel, 2004). La inmunización pasiva ha tenido un éxito parcial, debido a la poca uniformidad de anticuerpos maternales y fallas en la vacunación; sin embargo, es el principal método usado para proteger a los pollitos en las primeras semanas de vida (Saif, 1999).

Los programas de vacunación disponen de alternativas a través del uso de vacunas vivas, clasificadas como calientes, intermedias y suaves, de acuerdo a su capacidad para inducir daño en la bursa de aves SPF y la 
capacidad de infectar en presencia de niveles de anticuerpos altos, moderados o bajos, respectivamente. La necesidad de proteger a las aves de infecciones tempranas y la variación en la eficacia obtenida por vacunas convencionales ha hecho necesario que se desarrollen nuevas tecnologías en la protección contra la EIB; es así, que una nueva alternativa es el uso de la vacunación in ovo usando un complejo vacunal antígeno-anticuerpo, teniendo en cuenta que el pollo no eclosionado es inmunocompetente y puede desarrollar una respuesta inmune temprana y eficaz (Gagic et al., 1999; Whitfill et al., 2002). El objetivo del presente estudio fue evaluar la protección del Complejo Vacunal Antígeno-Anticuerpo administrado in ovo frente a un desafío experimental con la cepa F52/70 de la Enfermedad de Gumboro en pollos de carne.

\section{Materiales y Métodos}

\section{Lugar de Estudio}

El estudio se llevó a cabo en el galpón experimental e instalaciones del Laboratorio de Patología Aviar y en el Laboratorio de Patología de la Facultad de Medicina Veterinaria de la Universidad Nacional Mayor de San Marcos, Lima.

\section{Animales y Vacunas}

Se utilizaron 450 pollos BB de carne machos de la línea Cobb Vantres, distribuidos en forma uniforme en tres grupos experimentales y criados en ambientes separados. Las aves recibieron el mismo tipo de alimento y agua ad libitum en la etapa de crianza. El alimento cubrió los requerimientos nutricionales necesarios.

Se usaron vacunas comerciales a virus vivo contra la enfermedad de Gumboro, conteniendo las cepas tipo Lukert (intermedia suave), 2512 (intermedia intermedia) y el complejo vacunal antígeno-anticuerpo usando una cepa 2512 (intermedia fuerte).

\section{Procedimiento Metodológico}

El grupo A fue vacunado vía agua de bebida a los 9 días con la cepa intermedia suave tipo Lukert y revacunados a los 19 días con la cepa intermedia intermedia 2512; el grupo B fue vacunado vía in ovo a los 18 días de incubación con aguja calibre 20 y 0.1 ?1 del inóculo en la cavidad amniótica con el complejo vacunal antígeno-anticuerpo; y el grupo $\mathrm{C}$ quedó como control no vacunado.

Al día 35 de edad, 20 aves de cada grupo se colocaron en un mismo ambiente y fueron desafiados con la cepa F52/70, vía ocu$\operatorname{lar}(60 \mu \mathrm{L} / \mathrm{dosis})$. Al tercer día post desafío se sacrificó y realizó la necropsia a 20 animales de cada grupo para determinar lesiones macroscópicas (edema y hemorragia) y microscópicas. Los signos clínicos (depresión y diarrea) y la mortalidad fueron evaluados durante los 3 días post desafío.

\section{Evaluación Macroscópica}

Se sacrificaron 12 aves de cada grupo por semana hasta los 47 días de edad, para evaluar el estado de la bursa y determinar el índice bursal [(Peso de la bursa / Peso corporal del ave) x 1000], según Giambrone (1987), donde:

$$
\begin{aligned}
& ? \quad 1.5-3.5=\text { Bursa normal } \\
& ? \quad 0.5-1.5=\text { Atrofia bursal } \\
& ? \quad \leq 0.5=\text { Severa atrofia bursal }
\end{aligned}
$$

\section{Evaluación Microscópica}

Se tomaron 5 muestras de bursa al azar de las aves sacrificadas semanalmente y post desafío para la evaluación microscópica. Se hizo una valoración cualitativa de las lesiones de la bursa a nivel del epitelio de las plicas, folículos linfoides y tejido conectivo, empleando una calificación de daño bursal de 1 a 4 (adaptado de Mohamed et al., 1996 y Skeeles, 2001): 


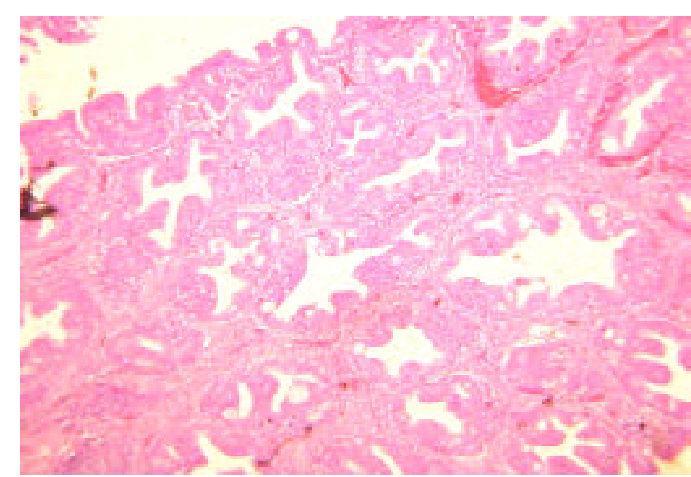

(a)

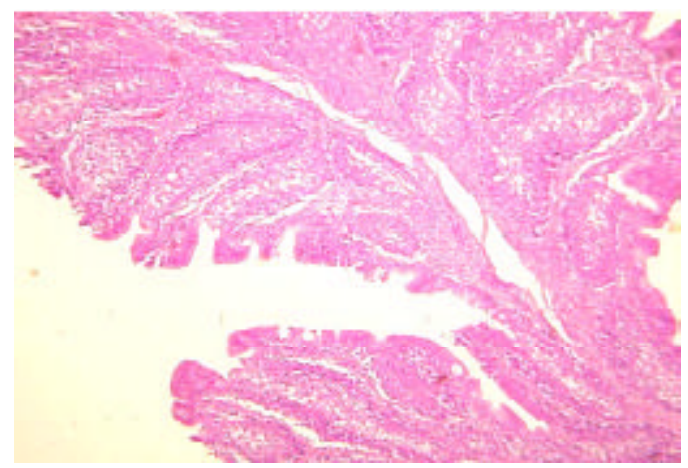

(b)

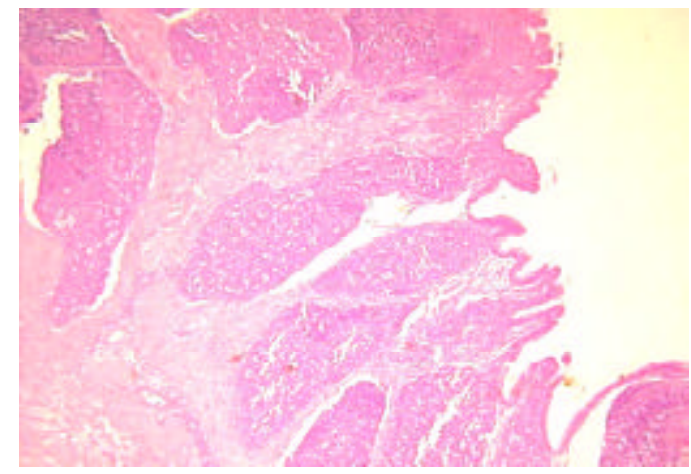

(c)

Figura 1. Lesiones microscópicas en pollos de carne a los 3 días post desafío con la cepa F52/ 70. (a) Formaciones quísticas y hemorragias en el tejido bursal de aves del grupo A. (b) Moderada depleción linfoide y presencia de repliegues del epitelio de revestimiento (flechas) en bursas de aves del grupo B. (c) Severa depleción linfoide, necrosis y severa fibroplasia (flechas).

? $\quad$ Grado 1 = No hay lesiones (sin cambios significativos en el tejido bursal)

? Grado 2 = Existe poca depleción de células linfoides en pocos folículos (30\%)

? Grado 3 = Hay una moderada atrofia y depleción de células de los folículos (31$75 \%$ )

? $\quad$ Grado $4=$ Severa necrosis y atrofia en todos los folículos (>75\%)

El valor final de las lesiones bursales de cada grupo se obtuvo sacando el promedio de las calificaciones de las bursas evaluadas.

\section{Evaluación Serológica}

Se tomaron muestras de sangre vía punción alar para la evaluación de los títulos de anticuerpos contra el virus de EIB de 25 aves por grupo al día 1, 7, 14, 21, 28, 35, 42 y 47, mediante la prueba de ELISA con un kit comercial (IDEXX).

\section{Análisis Estadístico}

Se utilizó el análisis de varianza con un diseño al azar con tres tratamientos para evaluar el índice bursal y los niveles de anticuerpos entre los grupos experimentales. 


\section{Resultados}

Los signos clínicos observados entre las 24 y 72 horas post desafío en los grupos experimentales se caracterizaron por la presencia de diarrea y depresión. El grupo C, no vacunado, presentó el mayor porcentaje de diarrea $(70 \%)$ y depresión (62\%) post desafío. No se observó mortalidad en ninguno de los grupos (Cuadro 1).

Las lesiones macroscópicas en las aves desafiadas se caracterizaron por la presentación de hemorragias y edema con presencia de material gelatinoso. Las aves del grupo $\mathrm{C}$ presentaron el mayor porcentaje de lesiones agudas (75\% con edema y $40 \%$ con hemorragias) (Cuadro 1).

Las aves desafiadas de los grupos A y $\mathrm{C}$ presentaron lesiones más severas de bursa (3.5 y 3.1, respectivamente) en comparación al grupo B desafiado (2.2). Las lesiones histológicas se caracterizaron por depleción linfoide, hiperplasia, fibroplasia y formación de quistes (Fig. 1).

En las aves no desafiadas se observó que el grupo B presentó el mayor grado de lesión bursal durante los primeros 28 días, en comparación a los grupos A y C (Cuadro 2). El grupo A presentó las lesiones más severas a los 35 y 47 días.

Los valores de índice bursal obtenidos durante las primeras cuatro semanas, en los tres grupos experimentales, correspondieron a una calificación de bursa normal; presentando atrofia a partir de la quinta semana de edad en el grupo A (Cuadro 2). Los niveles de anticuerpos maternales al primer día de edad fueron similares entre los grupos experimentales. Los valores fueron disminuyendo con el tiempo, pero en los grupos A y B se apreció un ligero incremento a los 35 días de edad; sin embargo, solo en el grupo A hubo un incremento significativo en las dos últimas semanas (Cuadro 3).
Los signos clínicos observados en las aves desafiadas se caracterizaron por la presentación de depresión y diarrea, siendo más severos en el grupo control. Signos clínicos similares son descritos en la literatura, además de otros como anorexia, postración y deshidratación que ocurre en pollos de 3 a 6 semanas de edad (Lukert y Saif, 2003). No hubo casos de mortalidad por Gumboro, lo cual concuerda con otros estudios utilizando la misma cepa de desafío (Cardoso, 1999; Rautenschlein et al., 2003; Pérez, 2005). Otros estudios empleando la vacunación in ovo para EIB presentaron signos clínicos similares o más leves a los descritos en el presente estudio (Coletti et al., 2001; Corley et $a l .$, 2002). No obstante, la presentación y severidad de los signos clínicos se encuentran asociadas al grado de protección de las aves y la patogenicidad de la cepa de desafío (Banda y Villegas, 2002; Hafez, 2003; Lukert y Saif, 2003).

No se observaron lesiones macroscópicas al momento de la necropsia en el grupo B vacunado con el complejo antígeno-anticuerpo; sin embargo, en la evaluación microscópica de las bursas de las aves desafiadas se observaron lesiones significativas de grado 2 a 3, especialmente en los grupos A y C. Hallazgos similares fueron encontrados por Pérez (2005) en aves desafiadas a los 32 días de edad con la cepa F52/70. Asimismo, los trabajos realizados por Al Natour et al. (2004) empleando cepas vacunales intermedias y variantes frente a desafíos experimentales entre la $2^{\mathrm{a}}$ y $5^{\mathrm{a}}$ semana de edad describen lesiones similares, pero con una mayor severidad en relación a la exposición más temprana de la cepa de desafío. Por otro lado, Giambrone et al. (2001), utilizando la técnica de la vacunación in ovo para la evaluación de cepas intermedias, observaron que solo un $13 \%$ de aves fue susceptible al desafío de una cepa estándar, mientras que el $40 \%$ fue susceptible al desafío de una cepa variante de la EIB. 
Cuadro 1. Pollos de carne ${ }^{1}$ machos desafiados con la cepa F52/70: mortalidad y signos clínicos entre las 24 y 72 horas post desafío y lesiones macroscópicas de la bursa a los 3 días post desafío, expresado en porcentaje de ocurrencia

\begin{tabular}{llccc}
\hline & & Grupo A & Grupo B & Grupo C \\
\hline Mortalidad & & 0 & 0 & 0 \\
\multirow{2}{*}{ Signos clínicos } & Depresión & 30 & 25 & 62 \\
& Diarrea & 32 & 25 & 70 \\
\multirow{3}{*}{ Lesiones en bursa } & Edema & 15 & 0 & 75 \\
& Hemorragia & 20 & 0 & 40 \\
\hline
\end{tabular}

1 Grupo A: vacunado a los 9 días con la cepa tipo Lukert y revacunados a los 19 días con la cepa 2512; Grupo B: fue vacunado vía in ovo a los 18 días de incubación

Cuadro 2. Lesiones microscópicas en la bursa e índice bursal en pollos de carne vacunados contra la Enfermedad de Gumboro

\begin{tabular}{lcccccccc}
\hline \multirow{2}{*}{ Grupo $^{1}$} & & \multicolumn{7}{c}{ Edad (días) } \\
\cline { 2 - 9 } & & 7 & 14 & 21 & 28 & 35 & 42 & 47 \\
\hline \multirow{2}{*}{ Lesiones en $_{\text {bursa }^{2}}$} & $\mathrm{~A}$ & 1.32 & 1.56 & 1.18 & 1.75 & 3.26 & 2.52 & 3.73 \\
& $\mathrm{~B}$ & 2.12 & 2.25 & 2.32 & 2.53 & 2.16 & 3.10 & 2.16 \\
& $\mathrm{C}$ & 1 & 1.5 & 1.25 & 1.15 & 1.51 & 2 & 3.11 \\
Índice bursal $^{3}$ & $\mathrm{~A}$ & 1.97 & 2.09 & 2.49 & 2.48 & 1.07 & 0.56 & 0.54 \\
& $\mathrm{~B}$ & 1.96 & 1.67 & 2.12 & 1.83 & 1.71 & 1.71 & 1.78 \\
& $\mathrm{C}$ & 1.70 & 1.93 & 2.78 & 2.25 & 2.22 & 2.16 & 2.16 \\
\hline
\end{tabular}

${ }^{1}$ Grupo A: vacunado a los 9 días con la cepa tipo Lukert y revacunados a los 19 días con la cepa 2512; Grupo B: vacunado vía in ovo a los 18 días de incubación con el complejo vacunal antígenoanticuerpo; Grupo C: control no vacunado

2 Adaptado de Mohamed et al. (1996) y Skeeles (2001)

3 Según Giambrone (1987): 1.5-3.5 = bursa normal; 0.5-1.5 = atrofia bursal; 0.5 = severa atrofia bursal

Cuadro 3. Niveles de anticuerpos (PGT) en pollos de carne ${ }^{1}$ vacunados contra la Enfermedad de Gumboro pero sin desafío experimental

\begin{tabular}{lcccccccc}
\hline Edad (días) & 1 & 7 & 14 & 21 & 28 & 35 & 42 & 47 \\
\hline Grupo A & 4954 & 2448 & 1714 & 448 & 286 & 477 & 2798 & 2123 \\
Grupo B & 5332 & 2357 & 1377 & 620 & 211 & 429 & 205 & 175 \\
Grupo C & 4954 & 2748 & 1410 & 481 & 170 & 115 & 49 & 76 \\
\hline
\end{tabular}

${ }^{1}$ Grupo A: vacunado a los 9 días con la cepa tipo Lukert y revacunados a los 19 días con la cepa 2512; Grupo B: vacunado vía in ovo a los 18 días de incubación con el complejo vacunal antígenoanticuerpo; Grupo C: control no vacunado

PGT: Promedio Geométrico Total 
En las primeras semanas de edad, el índice bursal fue incrementándose hasta llegar a sus valores máximos en la $4^{\text {ta }}$ semana, desarrollo considerado como normal (Giambrone, 1987). La atrofia bursal observada en la $5^{\text {a }}$ semana es similar a la observada en otros estudios en aves vacunadas con la cepas intermedias 2512 (Gallardo, 1998), aunque hay otros estudios que demuestran una tendencia a la atrofia a la $4^{\text {ta }}$ semana de edad (Winterfield et al., 1981). Asimismo, Colletti et al. (2001) y Corley et al. (2002), empleando complejos vacunales antígenoanticuerpo para la enfermedad de Gumboro, describen valores ligeramente menores a los 28 y 35 días de edad, pero con calificación de bursa normal; sin embargo, Haddad et al. (1997) y Whitfill et al. (2002), reportan valores de índice bursal de 1.01 y 0.82 , compatibles con atrofia, a los 31 y 35 días de edad, respectivamente. Estas diferencias están relacionadas al momento de replicación del virus vacunal en la bursa, la patogenicidad de la cepa vacunal, la vía de administración, la manipulación y las características de aislamiento de la cepa.

La evaluación histopatológica muestra que el grupo B presentó un mayor grado de lesiones bursales durante las primeras cuatro semanas en comparación al grupo A, lo cual se puede asociar a una constante actividad del virus vacunal. Winterfield et al. (1981) describen lesiones de ligera a moderada intensidad producidos por cepas vacunales 2512. Asimismo, Giambrone et al. (2001) encontraron que aves vacunadas in ovo con cepas intermedias mostraron severo daño bursal a partir de la $1^{\mathrm{a}}$ y $3^{\mathrm{a}}$ semana de edad en aves SPF y con anticuerpos maternales, respectivamente. Similares resultados se obtuvieron en pollos comerciales vacunados con el complejo vacunal antígeno-anticuerpo (Haddad et al., 1997; Gagic et al., 1999).

Los anticuerpos maternales fueron disminuyendo hasta la $4^{\mathrm{a}}$ semana, momento en que aun no se observaba la seroconversión de los grupos vacunados. El incremento de los niveles de anticuerpos a los 35 días de edad en los grupos A y B se puede relacionar con una respuesta vacunal; sin embargo, en el grupo B se aprecia que no hubo una buena seroconversión, cayendo los niveles de anticuerpos hasta un PGT de 175 en comparación al PGT de 2123 del grupo A. Similares resultados fueron obtenidos por Corley et al. (2001) en pollos comerciales no desafiados vacunados in ovo con el complejo vacunal antígeno-anticuerpo y revacunado con la cepa 2512. Asimismo, en aves desafiadas a los 21 días de edad, vacunadas in ovo con una cepa intermedia, se observaron resultados similares con niveles de anticuerpos menores a 500 (Coletti et al., 2001).

Dado que el mecanismo de acción de los complejos vacunales antígeno-anticuerpo de la EIB no se encuentra totalmente esclarecido, se puede determinar a partir de los resultados obtenidos en el presente trabajo que la protección observada en el grupo A estaría relacionada a una estimulación activa de la respuesta inmune mediada por células o al mecanismo de acción sobre los centros germinales de la bursa y otros órganos linfoides, con una participación activa de macrófagos, células reticulares y células dendríticas que responden a receptores de la vía del complemento y fracción Fc de membrana.

\section{ConClusiones}

? Los dos programas de vacunación indujeron una buena protección frente al desafío con la cepa F52/70 de la enfermedad de Gumboro, en comparación con el grupo control no vacunado, donde se observaron signos clínicos y lesiones bursales más severos.

? El programa de la vacunación in ovo, empleando el complejo vacunal antígenoanticuerpo, parece ser seguro, eficaz y conveniente para la avicultura, porque presentó una mejor protección para la bursa que el programa usando vacunas intermedias. 


\section{LITERATURA CITADA}

1. Al Natour MQ, Ward LA, Saif YM, Brown BS, Keck KL. 2004. Effect of diferent levels of maternally derived antibodies on protection against Infectious Bursal Disesase virus. Avian Dis 48: 177-182.

2. Banda A, Villegas P. 2002. Enfermedad infecciosa de la bolsa. Industria Avícola, Colombia 49(7): 22-25.

3. Cardozo BL. 1999. Estudio clínico del sistema inmunocompetente en tres parvadas de pollo de engorda. En: IV Jornada Médico Avícola. México DF: Facultad de Medicina Veterinaria y Zootecnia, Universidad Nacional Autónoma de México.

4. Coletti M, Rossi ED, Franciosini MP, Pasamonti F, Taconi G, Marini C. 2001. Efficacy and safety of an Infectious Bursal disease virus intermediate vaccine in ovo. Avian Dis 45:1036-1043.

5. Corley M, Giambrone J, Dormitorio T. 2001. Detection of infectious bursal disease vaccine viruses lymphoid tissues In ovo vaccination of specific-pathogenfree-embryos. Avian Dis 45: 897-905.

6. Corley M, Giambrone J, Dormitorio T. 2002. Evaluation of the immune response and detection of Infectious Bursal Disease viruses by reverse transcriptase polimerase (RTP) and ELISA in ovo vaccination. Avian Dis 46: 803-809.

7. Gagic M, Hill S, Sharma J. 1999. In ovo vaccination of specific pathogen free chickens with vaccines containing multiple agents. Avian Dis 43: 293-301.

8. Gallardo WL. 1998. Evaluación serológica e histopatológica de pollos broilers vacunados contra la enfermedad de Gumboro a los doce días de edad. Tesis de Médico Veterinario. Lima: Facultad de Medicina Veterinaria, Univ Nacional Mayor de San Marcos. 54 p.

9. Giambrone J. 1987. Evaluación y relaciones morfométricas en la enferme- dad infecciosa de la Bursa como método de diagnóstico. The American Association of Avian Pathology. Georgia, USA. Bull Tech 23: 24-38.

10. Giambrone T, Dormitorio T, Brown T. 2001. Safety and efficacy of in ovo administration of Infectious Bursal Disease viral vaccines. Avian Dis 45: 144-148.

11. HadadE, Whitfill C, Aviakan A, Ricks C, Andrews P, Thoma J, Wakenel P. 1997. Efficacy of a novel Infections Bursal Disease virus immune complex vaccine in broiler chickens. Avian Dis 41: 882-889.

12. Hafez H. 2003. Infección de la bolsa de Fabricio. Evolución de la enfermedad de Gumboro e innovaciones para su control. En: XIV Curso de Actualización Avimex. México DF.

13. Lukert PD, Saif YM. 2003. Infectious Bursal Disease. In: Calnek BW, Beard CW, Yoder HW, Reid WH, Barnes HJ (eds). Diseases of poultry. $11^{\text {th }}$ ed. Iowa: Iowa State University Press. p 161-174.

14. Montiel 2004. Interacciones entre agentes inmunosupresores: Anemia Infecciosa Aviar, Gumboro y Marek. En: II Seminario Internacional AMEVEA PERÚ. Lima: Asociación de Médicos Veterinarios Especialistas en Aves.

15. Mohamed K, Al-Natour M, Ward L, Saif Y. 1996. Pathogenicity, attenuation, and immunogenicity of infectious bursal disease virus. Avian Dis 40: 567-571.

16. Pérez CM. 2005. Evaluación de dos programas de vacunación que contienen la cepa 2512 de la enfermedad de Gumboro frente a la infección experimental con la cepa F52/70. Tesis de Médico Veterinario. Lima: Facultad de Medicina Veterinaria, Univ Nacional Mayor de San Marcos. $60 \mathrm{p}$.

17. Rautenschlein S, Hung-Yueh Y, Sharma J. 2003. Comparative immunopathogenesis of mild, intermediate, and virulent strains of classic Infectious Bursal Disease virus. Avian Dis 47: 66-78.

18. Rosales G. 2000. Control actual de la Enfermedad Infecciosa de la Bursa. En: 
Memorias XI Seminario Internacional de Patología Aviar. Georgia, USA. p 468-481.

19. Saif YM. 1999. Control y prevención de la Enfermedad Infecciosa de la Bursa. En: XVI Congreso Latinoamericano de Avicultura. Maracaibo, Venezuela.

20. Skeeles B. 2001. Score de lesiones bursales. Laboratorios Shering-Plough. EEUU. Boletín Informativo. 2 p.
21. Winterfield R, Dhillo, A, Thacke H. 1981. Characteristics of apparent derivatives of the 2512 strain of infectious bursal disease virus when used as vaccines. Avian Dis 25: 900-910.

22. Whitfill C, Aviakan A, Haddad E, Wakenel P. 2002. Vacunas con el complejo virus-anticuerpo: Presente y futuro. Avicultura Profesional 20(1): 17-22. 